\title{
SCALABILITY OF PEER CONFIGURATION MANAGEMENT IN PARTIALLY RELIABLE AND AD HOC NETWORKS
}

\author{
Mark Burgess \\ Faculty of Engineering, Oslo University College, Norway \\ Mark.Burgess@iu.hio.no \\ Geoffrey Canright \\ Telenor Research, Fornebu, Oslo, Norway \\ Geoffrey.Canright@telenor.com
}

\begin{abstract}
Current interest in ad hoc and peer-to-peer networking technologies prompts a re-examination of models for configuration management, within these frameworks. In the future, network management methods may have to scale to millions of nodes within a single organization, with complex social constraints. In this paper, we discuss whether it is possible to manage the configuration of large numbers of network devices using well-known and no-so-well-known configuration models, and we discuss how the special characteristics of ad hoc and peer-to-peer networks are reflected in this problem.
\end{abstract}

Keywords: Configuration management, ad hoc networks, peer to peer.

\section{Introduction}

Configuration management is about ensuring that the operational state of a device or host conforms to specifications lain down by a site policy. The configuration of a host ensures its efficiency, correctness and security in performing its function. System configuration is usually a specification of file or database contents, attributes, and process or service characteristics, including access rights, software customization and so on. A number of approaches has been devised for configuration management. For instance, the IETF model of configuration management revolves traditionally around the Simple Network Management Protocol (SNMP)[6]. This is read/write state based protocol for altering values in a management information database (MIB), and is used by a number of commercial software products. The 'Telecommunications Management Network' or TMN[12] is an alternative scheme designed for telecommunications networks and has a strong relationship with the OSI management model. These systems use an abstraction based on the concept of 'managed objects'. An different approach is used by systems like cfengine[2] and PIKT[13], which use descriptive languages to describe the attributes of many objects at the same time, and agents to enforce the rules.

The ability to send or receive messages is crucial to configuration management of network devices and hosts. Indeed, maintaining the configuration of hosts over time has many features in common with the problem of information transmission over a noisy channel[5]. Today, distributed systems sport a global geography, and are linked,

The original version of this chapter was revised: The copyright line was incorrect. This has been corrected. The Erratum to this chapter is available at DOI: 10.1007/978-0-387-35674-7_66 
both conceptually and physically, by a network infra-structure. Passing messages from one part of a system to another is subject to a plethora of uncertainties. For example, SNMP uses an unreliable transport protocol UDP for communication; any configuration scheme that relies on the availability of a resource or component at a specific moment has only a limited chance of being carried out. Systems can be unavailable due to power failures, physical breakages, absence of dependencies and so on. There is thus an ad hoc element to network connectivity even in an ostensibly permanent infrastructure. The additional complication of mobile services, with partial or intermittent connectivity adds to this problem.

An 'ad hoc' network (AHN) is defined to be a networked collection of mobile hosts, each of which has the possibility to route information. The union of those hosts forms an arbitrary graph that changes with time. The nodes are free to move randomly; thus the network topology may change rapidly and unpredictably. Clearly ad hoc networks are important in a mobile computing environment, where hosts are partially or intermittently connected to other hosts. While there has been some discussion of de-centralized network management using mobile agents[15], the problem of mobile nodes (and so strongly time-varying topology) has received little attention. However, we will argue below that ad-hoc networks provide a useful framework for discussing the problems surrounding configuration management in all network types, both fixed and mobile. This should not be confused with the notion of 'ad hoc management'[11], which concerns randomly motivated and scheduled checks of the hosts.

The plan for our paper is as follows. We begin by outlining how reliability can be discussed in terms of ad hoc connectivity in order to take advantage of its known scaling properties. Then noting how peer to peer communication implies decentralized policy, we estimate the required flow of configuration information as a function of the number of hosts, for a number of management models, in order to determine their scalability.

\section{Availability of peers in a network}

The probability that a host will be correctly configured is related to reliability of its communication with a policy source. As a simplest case, we assume that the reliability of each node and each link is independent of all others, so that the probabilities of availability are all independent random variables. In general this is not true, since some hosts/nodes depend on others for crucial services (e.g. the domain name service (DNS)), but this should suffice to gauge orders of magnitude.

DEFINITION 1 A set of nodes or hosts is defined by a vector of probabilities $\vec{h}^{T}=$ $\left(p_{1}, p_{2}, \ldots, p_{N}\right)$, where $p_{i}(i=1 \ldots N)$ is the probability that node $i$ is available. If the probabilities are 1, the hosts are said to be reliable, otherwise they are partially reliable.

The nodes themselves may have any geographical location, and may be connected by any means. The connectivity between the nodes is represented by a matrix.

DEFINITION 2 A network is defined by its adjacency matrix. By convention, the adjacency matrix of a network or graph is a symmetric matrix with zero leading diagonal. Zeroes denote no connectivity, while a 1 means a connection. The notation A(1) distinguishes this (instantaneous) matrix, whose entries are binary-valued, from the time-averaged matrix discussed below. Owing to access and routing controls, this matrix need not be symmetrical in practice, but we shall not address that issue here. 
The properties of networks can be discussed in detail, using the adjacency matrix representation (see for instance, ref. [14]). It is not our intention to go into excessive detail here, but rather to distill a way of estimating the properties of networks. For this, we choose to look at the average properties of the networks.

We define a simple measure of the availability of a service, transmitted within a closed network, by an invariant scalar value $\chi$ :

DEFINITION 3 The connectivity, $\chi$, of a network $\mathcal{N}$, is the probability (averaged over all pairs of nodes) that a message can be passed directly between any two nodes. $\chi$ may be written as

$$
\chi=\frac{1}{N(N-1)} \vec{h}^{\mathrm{T}} A \vec{h} .
$$

$\chi$ has a maximum value of 1 , when every node is connected to every other, and a minimum value of zero when all nodes are disconnected.

For a fixed topology and time-independent node availabilities, $\chi$ is a constant characterizing the network. In general $\chi$ is time-dependent; one then obtains a static figure for the network by taking the long-time average.

$$
\langle\chi\rangle=\lim _{n \rightarrow \infty} \frac{1}{n} \sum_{i=1}^{n} \chi\left(t_{i}\right) .
$$

The utility of this measure is that it enables us to gauge and compare different network configurations on equal terms. It is also the vehicle by which we can map the problem of unreliable hosts in a fixed network onto a corresponding problem of reliable hosts in an ad hoc network.

\section{Ad hoc networks}

Ad hoc networks are networks whose adjacency matrices are subject to a strong, apparently random time variation. If we look at the average adjacency matrices, over time, then we can represent the probability of connectivity in the network as an adjacency matrix of probabilities.

DEFINITION 4 An ad hoc network is represented by a symmetric matrix of probabilities for adjacency. Thus the time average of the adjacency matrix (for, e.g., four nodes) may be written as

$$
\langle A\rangle=\left(\begin{array}{cccc}
0 & p_{12} & p_{13} & p_{14} \\
p_{21} & 0 & p_{23} & p_{24} \\
p_{31} & p_{32} & 0 & p_{34} \\
p_{41} & p_{42} & p_{43} & 0
\end{array}\right)
$$

An ad hoc network is therefore a partially reliable network.

To motivate our discussion further, we note that:

THEOREM 1 A fixed network of partially-reliable nodes, $h_{i}$, is equivalent to an ad hoc network of reliable nodes, on average. 
ProOF 1 This is easily seen from the definition of the connectivity, using a matrix component form:

$$
\begin{aligned}
N(N-1)\langle\chi\rangle & =\sum_{i j} h_{i}\left(p_{i}\right)\left\langle A_{i j}(1)\right\rangle h_{j}\left(p_{j}\right) \\
& =\sum_{i j} h_{i}(1)\left\langle A_{i j}\left(p_{i} p_{j}\right)\right\rangle h_{j}(1) .
\end{aligned}
$$

This concludes the proof.

The proof demonstrates the fact that one can move the probabilities (uncertainties) for availability from the host vectors to the connectivity matrix and vice versa; for example

$$
\left(\begin{array}{l}
p_{1} \\
p_{2} \\
p_{3}
\end{array}\right)^{\mathrm{T}}\left(\begin{array}{lll}
0 & 1 & 1 \\
1 & 0 & 1 \\
1 & 1 & 0
\end{array}\right)\left(\begin{array}{l}
p_{1} \\
p_{2} \\
p_{3}
\end{array}\right)=\left(\begin{array}{l}
1 \\
1 \\
1
\end{array}\right)^{\mathrm{T}}\left(\begin{array}{ccc}
0 & p_{1} p_{2} & p_{1} p_{3} \\
p_{2} p_{1} & 0 & p_{2} p_{3} \\
p_{3} p_{1} & p_{3} p_{2} & 0
\end{array}\right)\left(\begin{array}{l}
1 \\
1 \\
1
\end{array}\right) .
$$

Thus an array of hosts with reliability probabilities $p_{i}$, is equivalent to an array of reliable hosts in an unreliable network, where the probability of communication between them is the product of probabilities (assumed independent) from the reliability vector.

Superposed onto the routing problem is another problem of conceptual dependence. One is not merely dependent on connectivity to provide a route for messages, but one depends on trusted sources of information. Thus the arrows from source to receiver are not merely bytes exchanged but authorized policy instructions. We shall consider this issue below.

\section{Peer to peer}

The emergence of network file sharing applications such as Napster and Gnutella has focused attention on an architecture known as peer-to-peer, whose aim is to provide worldwide access to information via a highly de-centralized network of 'peers'.

DEFINITION 5 A peer to peer network service is one in which each node, at its own option, participates in or abstains from exchanging data with other nodes, over a communications channel.

Peer to peer has a deeper significance than ad hoc file sharing. It is about the demotion of a central authority, in response to the political wishes of those participating in the network. This is an issue directly analogous to the policies used for configuration management. In large organizations, i.e. large networks, we see a frequent dichotomy of interest:

- At the high level, one has specialized individuals who can paint policy in broad strokes, dealing with global issues such as software versions, common security issues, organizational resource management, and so on. Such issues can be made by software producers, system managers and network managers.

- At the local level, users are more specialized and have particular needs, which large scale managers cannot address. Centralized control is therefore only a 
partial strategy for success. It must be supplemented by local know-how, in response to local environmental issues. Managers at the level of centralized control have no knowledge of the needs of specialized groups, such as the physics department of a university, or the research department of a company. In terms of configuration policy, what is needed is the ability to accept the advice of higher authorities, but to disregard it where it fails to meet the needs of the local environment. This kind of authority delegation is not catered for by SNMP-like models. Policy based management attempts to rectify some of these issues[8].

What we find then is that there is another kind of networking going on: a social network, superimposed onto the technological one. The needs of small clusters of users override the broader strokes painted by wide area management. This is the need for a scaled approach to system management[3].

\section{Configuration management in ad hoc networks}

Configuration management deals with the problem of establishing and maintaining a policy conformant configuration on workstations and other hosts distributed around a network. Policy is usually a set of rules and specifications about the software and resources of each host, defined by a central authority and disseminated to the individual hosts either on demand, or by common update.

Configuration management relies on two main things: i) the availability of trusted resources to each networked host, including a policy $P$, and ii) the consistency of the configuration specified by that policy. In an unpredictable environment one has potentially several problems: Critical dependencies, including the policy itself, can become unavailable or out of date; trust relationships are less certain if hosts cannot verify one another's' identity, location or integrity. Thus security and verifiable control, within specified time limits, are at stake.

Even in a fixed infrastructure network, with only partial connectivity, the availability of the resources is open to uncertainty. This means that the ability to correctly disseminate policy configuration is open to uncertainty. The framework of ad hoc networks thus encompasses a number of issues and offers a framework for discussing configuration strategies in general. In recent times, there has been a move towards self-configuring networks. Discovery protocols like JINI have to deal with the ad hoc nature of networks, and the protocols themselves will need to take the uncertainties in topology into account. Today, most protocols assume a fixed infra-structure.

One question that has been posed in this connection is whether a peer to peer strategy, for disseminating configuration policy, could provide a way of spreading information quickly about the network. If that were the case, then the temporary unavailability of a node to a central resource would not necessarily imply its isolation from fresh, critical data. This kind of data distribution has been discussed before[7] in connection with the scalability of software distribution. On the down side, peer to peer reliance is clearly an open invitation to engage in malicious activity.

\section{Predictability and scaling}

As networks grow, some configuration strategies do not scale well. They continue to be used, however, by force of habit. We are interested in examining the scaling properties of different configuration management schemes, especially in the context of network models that look to the future of configuration management. 
We consider a number of cases, in order of decreasing centralization, or increasing delegation. Our basic 'constitutive' assumption is that there is a simple linear relationship between the probability of successful configuration and the rate(s) of communication with the policy- and enforcement-source(s). We look only at the coarsest averages over time, in order to determine the long-term behaviours of the models. We consider a change of configuration ("charge") $\Delta Q$ to be proportional to an average rate of information flow (current) $I$, over a time $\Delta t$; that is $\Delta Q=I \Delta t$. This equation is valid when $I$ represents the time-averaged flow over the interval. Since we are interested in the limiting behaviour for long times, this is sufficient for our needs.

Now we apply this simple picture to configuration management for dynamic networks. We take the point of view of a 'typical' or 'average' host. It generates error in its configuration at the (average) rate $I_{\mathrm{err}}$, and receives corrections at the rate $I_{\text {repair }}$. Hence the rate of increase of error for the average node is:

$$
I_{\text {fail }}=\left(I_{\text {err }}-I_{\text {repair }}\right) \theta\left(I_{\text {err }}-I_{\text {repair }}\right) .
$$

The Heaviside step-function is defined by $\theta(x)=1$ if $x>0$ and $\theta(x)=0$ if $x<=0$, and signifies the fact that, if the repair rate exceeds the error rate, then (on average, over long times) nothing remains outstanding and there is no net rise in configuration error. Thus this averaged quantity is never negative.

If random errors and changes to configuration occur at a rate $I_{\text {err }}$ and the configuration agent is unavailable to correct them, then $I_{\text {fail }}=I_{\text {err }}$. If this holds during a time $\Delta t$, the configuration falls behind by an amount:

$$
\underset{(\Delta Q)}{\text { Bytes }} \underset{\text { missing }}{\text { bytes } / \text { sec }} \underset{\left(I_{\text {err }}\right)}{\text { seconds }} \times \underset{(\Delta t)}{\text { unavailable }} .
$$

In the following we will use $p$ to denote the average (over time, and over all nodes) probability that configuration management information flow (repair current) is not available to a node. This unavailability may come from either link or node unreliability. We can lump all the unreliability into the links (see above) and so write $p=\left(1-\left\langle A_{i j}\right\rangle\right)$, where $\left\langle A_{i j}\right\rangle$ denotes both time and node-pair average. Each node then can only receive repair current during the fraction $(1-p)$ of the total elapsed time.

The repair current is generated by two possible sources in our models: i) a remote source, and ii) a local source. In each case, the policy can be transmitted and/or enforced at a maximum rate given by the channel capacity of the source. We shall denote the channel capacities by $C_{R}$ and $C_{L}$ for remote and local sources for clarity, but we assume that $C_{R} \sim C_{L}$, since source and target machines are often comparable, if not identical. If the communication by network acts as a throttle on these rates, then one can further assume that $C_{R}<C_{L}$. In any case, the weakest link determines the effective channel capacity. Note that in the case of a confluence of traffic, as in the star models below, the channel capacity will have to be shared by the incoming branches. We now have a criterion for eventual failure of a configuration strategy. If $I_{\text {fail }}=\frac{\Delta Q}{\Delta t}>0$, the average configuration error will grow monotonically for all time, and the system will eventually fail in continuous operation. Our strategy is then to look at the scaling behaviour of $I_{\text {fail }}$ as the number of nodes $N$ grows large. 
Table 1. Comparison of models from the viewpoint of the different dimensions: policy dissemination, enforcement, freedom of choice, whether hosts can exchange chosen policy ideas with peers and how political control flows. A 'push' model implies a forcible control policy, whereas 'pull' signifies the possibility to choose. Model 3 lies between these two, in having the possibility but not the inclination to choose.

\begin{tabular}{cccccc} 
Model & $\begin{array}{c}\text { Application } \\
\text { Topology }\end{array}$ & Enforcement & $\begin{array}{c}\text { Policy } \\
\text { Freedom }\end{array}$ & $\begin{array}{c}\text { Policy } \\
\text { Exchange }\end{array}$ & $\begin{array}{c}\text { Control } \\
\text { Structure }\end{array}$ \\
\hline \hline 1 & Star & Transmitted & No & No & Radial push \\
2 & Star & Transmitted & No & No & Radial push \\
3 & Mesh & Local & No & No & Radial pull \\
4 & Mesh & Local & Yes & No & Radial pull \\
5 & Mesh & Local & Yes & Yes & Hierarchical pull \\
6 & Mesh & Local & Yes & Yes & P2P pull
\end{tabular}

\section{Star model}

The traditional (idealized) model of host configuration is based on the idea of remote management (e.g. using SNMP). Here one has a central manager who decides and implements policy from a single location, and all networks and hosts are considered to be completely reliable. The manager must monitor the whole network, using bi-directional communication. This leads to an $N: 1$ ratio of clients to manager (see fig 1). This first model is an idealized case in which there is no unreliability in any

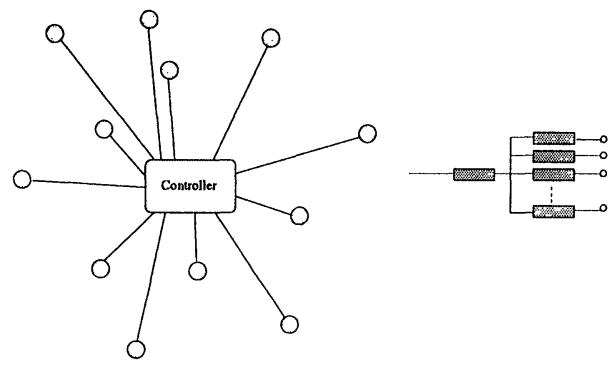

Figure 1 Model 1: the star network. A central manager maintains bi-directional communication with all clients. The links are perfectly reliable, and all enforcement responsibility lies with the central controlier.

component of the system. It serves as a point of reference.

The topology on the left hand side of fig 1 is equivalent to that on the right hand side. We can assume a flow conservation of messages on average, since any dropped packets can be absorbed into the probabilities for success that we attribute to the adjacency matrix. Thus the currents must obey Kirchoff's law:

$$
I_{\text {controller }}=I_{1}+I_{2}+\ldots I_{N} .
$$

The controller current cannot exceed its capacity, which we denote by $C_{S}$. We assume that the controller puts out repair current at its full capacity (since the Heaviside function corrects for lower demand), and that all nodes are average nodes. This gives that $I_{\text {repair }}=\frac{C_{S}}{N}$. The total current is limited only by the bottleneck of queued messages at the controller, thus the throughput per node is only $1 / N$ of the total capacity. We can now write down the failure rate in a straightforward manner:

$$
I_{\text {fail }}=\left(I_{\text {err }}-\frac{C_{S}}{N}\right) \theta\left(I_{\text {err }}-\frac{C_{S}}{N}\right) .
$$


As $N \rightarrow \infty, I_{\text {fail }} \rightarrow I_{\text {err }}$ - that is, the controller contributes a vanishing repair current per node. The system fails however at a finite $N=N_{\text {thresh }}=C_{S} / I_{\text {err }}$. This highlights the clear disadvantage of centralized control, namely the bottleneck in communication with the controller.

\section{Star model in intermittently connected environment}

The previous model was an idealization, and was mainly of interest for its simplicity. Realistic centralized management must take into account the unreliability of the environment.

In an environment with partially reliable links, a remote communication model bears the risk of not reaching every host. If hosts hear policy, they must accept and comply, if not, they fall behind in the schedule of configuration. Monitoring in distributed systems has been discussed in ref. [1].

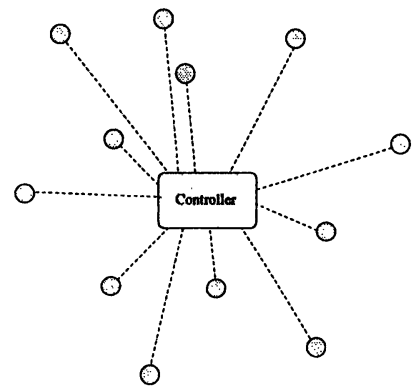

Figure 2 Model 2: a star model, with built-in unreliability. Enforcement is central as in Model 1.

The capacity of the central manager $C_{S}$ is now shared between the average number of hosts $\langle N\rangle$ that is available, thus

$$
I_{\text {repair }}=\frac{C_{S}}{N\left\langle A_{i j}\right\rangle} \equiv \frac{C}{\langle N\rangle} .
$$

This repair current can reach the host, and serve to decrease its policy error $\Delta Q$, during the fraction of time $(1-p)$ that the typical host is reachable. Hence we look at the net deficit $\Delta Q$ accrued over one "cycle" of time $\Delta t$, with no repair current for $p \Delta t$, and a maximal current $C_{S} /\langle N\rangle$ for a time $(1-p) \Delta t$. This deficit is then

$$
\Delta Q(\Delta t)=I_{\mathrm{err}} p t+\left(I_{\mathrm{err}}-\frac{C_{S}}{\langle N\rangle}\right)(1-p) \Delta t
$$

(here it is implicit that a negative $\Delta Q$ will be set to zero). Thus, the average failure rate is

$$
I_{\mathrm{fail}}=I_{\mathrm{err}} p+\left(I_{\mathrm{err}}-\frac{C_{S}}{\langle N\rangle}\right)(1-p)=I_{\mathrm{err}}-\frac{C_{S}}{N} .
$$

(Again there is an implicit $\theta$ function to keep the long-time average failure current positive.) This result is the same as for Model 1, the completely reliable star. This is because we assumed the controller was clever enough to find (with negligible overhead) those hosts that are available at any given time, and so to only attempt to communicate with them. 
This model then fails (perhaps surprisingly), on average, at the same threshold value for $N$ as does Model 1 . If the hunt for available nodes places a non-negligible burden on the controller capacity, then it fails at a lower threshold.

\section{Mesh topology with centralized policy and local enforcement}

The serialization of tasks in the previous models forces configuration 'requests' to queue up on the central controller. Rather than enforcing policy by issuing every instruction from the central source, it makes sense to download a summary of the policy to each host and empower the host itself to enforce it.

There is still a centrally determined policy for every host, but now each host carries the responsibility of configuring itself. There are thus two issues: i) the update of the policy and ii) the enforcement of the policy. A pull model for updating policy is advantageous here, because every host then has the option to obtain updates at a time convenient to itself, avoiding confluence contentions; moreover, if it fails to obtain the update, it can retry until it succeeds. We ask policy to contain a self-referential rule for updating itself.

The distinction made here between communication and enforcement is important, because it implies distinct types of failure, and two distinct failure metrics: i) distance of the locally understood policy from the latest version, and ii) distance of host configuration from the ideal policy configuration. In other words: i) communication failure, and ii) enforcement failure.

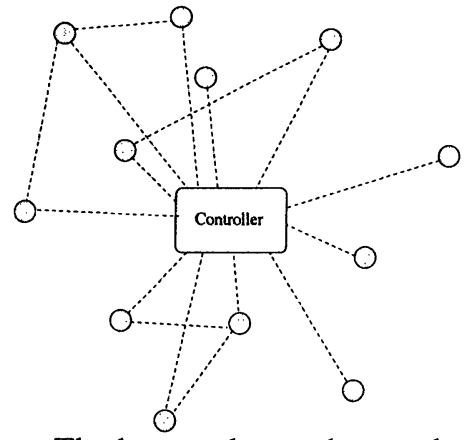

Figure 3 Model $3 . \quad$ Mesh topology. Nodes can learn the centrally-mandated policy from other nodes as well as from the controller. Since the mesh topology does not assure direct connection to the controller, each node is responsible for its own policy enforcement.

The host no longer has to share any bandwidth with its peers, unless it is updating its copy of the policy, and perhaps not even then, since policy is enforced locally and updates can be scheduled to avoid contention.

Let $I_{\text {update }}$ be the rate at which policy must be updated. This current is usually quite small compared to $I_{\mathrm{err}}$, and was neglected in the previous models. Based on the two failure mechanisms present here, we break up the failure current into two pieces: $I_{\text {fail }}=I_{\text {fail }}(i)+I_{\text {fail }}(i i)$. The former term is

$$
I_{\text {fail }}(i)=\left(I_{\text {err }}-C_{L}\right) \theta\left(I_{\mathrm{err}}-C_{L}\right) ;
$$

this term is independent of $N$ and may be made zero by design. $I_{\text {fail }}(i i)$ is still determined by the ability of the controller to convey policy information to the hosts. However, the load on the controller is much smaller since $I_{\text {update }} \ll I_{\text {err }}$. Also, the topology is a mesh topology. In this case the nodes can cooperate in diffusing policy updates, via flooding (Note, flooding in the low-level sense of a datagram multicast is not necessarily required, but the effective dissemination of the policy around the network is an application layer flood.) . 
The worst case-in which the hosts compete for bandwidth, and do not use flooding over the mesh-is that, for large $N, I_{\text {fail }} \rightarrow I_{\text {update. }}$. This is a great improvement over the two previous models, since $I_{\text {update }} \ll I_{\text {err }}$. However note that this can be further improved upon by allowing flooding of updates: the authorized policy instruction can be available from any number of redundant sources, even though the copies originate from a central location. In this case, the model truly scales without limit, i.e. $I_{\text {fail }}=0$.

There is one caveat to this encouraging result. If the (meshed) network of hosts is truly an ad-hoc network of mobile nodes, employing wireless links, then connections are not feasible beyond a given physical range $r$. In other words, there are no longrange links: no links whose range can grow with the size of the network. As a result of this, if the AHN grows large (at fixed node density), the path length (in hops) between any node and the controller scales as a constant times $\sqrt{N}$. This growth in path length limits the effective throughput capacity between node and controller, in a way analogous to the internode capacity. The latter scales as $1 / \sqrt{N}[9,10]$. Hence, for sufficiently large $N$, the controller and AHN will fail collectively to convey updates to the net. This failure will occur at a threshold value defined by

$$
I_{\text {fail }}(i i)=I_{\text {update }}-\frac{C_{S}}{c \sqrt{N_{\text {thresh }}}}=0
$$

where $c$ is a constant. The maximal network size $N_{\text {thresh }}$ is in this case proportional to $\left(\frac{C_{S}}{I_{\text {update }}}\right)^{2}$-still considerably larger than for Models 1 and 2.

\section{Mesh topology with partial host autonomy and local enforcement}

As a variation on the previous model, we can begin to take seriously the idea of distance from a political centre. In this model, hosts can choose not to receive policy from a central authority, if it conflicts with local interests. Communication thus takes the role of conveying 'suggestions' from the central authority, in the form of the latest version of the policy. For instance, the central authority might suggest a new version of widely-used software, but the the local authority might delay the upgrade due to compatibility problems with local hardware. Local enforcement is now employed by each node to hold to its chosen policy $P_{i}$. Thus communication and enforcement use distinct channels (as with Model 3); the difference is that each node has its own target policy $P_{i}$ which it must enforce.

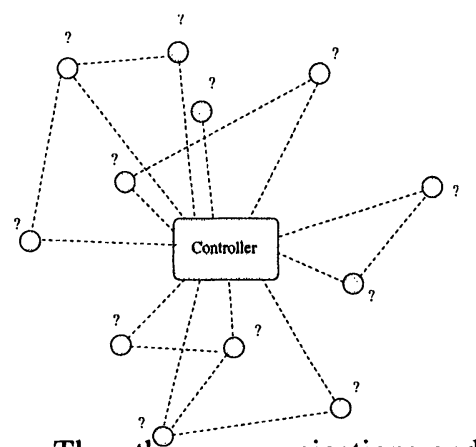

Figure 4 Model 4. As in Model 3 , except the hosts can choose to disregard or replace aspects of policy at their option. Question marks indicate a freedom of hosts to choose.

Thus the communications and enforcement challenges faced by Model 4 are the same (in terms of scaling properties) as for Model 3: i.e. $I_{\text {fail }}$ is the same as that in 
Model 3. Hence this model can in principle work to arbitrarily large $N$. Model 4 is the model used by cfengine[2,4]. The largest current clusters sharing a common policy are known to be of order $10^{4}$ hosts, but this could soon be of order $10^{6}$, with the proliferation of mobile and embedded devices.

\section{Mesh, with partial autonomy and hierarchical coalition}

An embellishment of Model 4 is to allow local groups of hosts to form policy coalitions, that serve to their advantage. Such groups of hosts might belong to one department of an organization, or to a project team, of even to a group of friends in a mobile network. Once groups form, it is natural to allow sub-groups and thence a generalized hierarchy of policy refinement through specialized social groups.

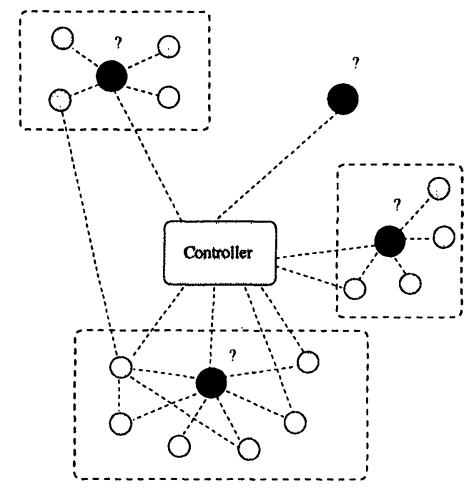

Figure 5 Model 5. Communication over a mesh topology, with policy choice made hierarchically. Sub-controllers (dark nodes) edit policy as received from the central controller, and pass the result to members of the local group (as indicated by dashed boxes). Question marks indicate the freedom of the controllers to edit policy from above.

If policies are public then the scaling argument of Model 3 still applies since any host could cache any policy; but now a complete policy must be assembled from several sources. Once can thus imagine using this model to distribute policy so as to avoid contention in bottlenecks, since load is automatically spread over multiple servers. In effect, by delegating local policy (and keeping a minimal central policy) the central source is protected from maximal loading. Specifically, if there are $S$ sub-controllers (and a single-layer hierarchy), then the effective update capacity is multiplied by $S$. Hence the threshold $N_{\text {thresh }}$ is multiplied (with respect to that for Model 3) by the same factor. This model could be implemented using cfengine, with some creative scripting.

\section{Mesh, with partial autonomy and inter-peer policy exchange}

The final step in increasing autonomy is the free exchange of information between arbitrary hosts. Hosts can now offer one another information, policy or source materials in accordance with an appropriate trust model. In doing so, impromptu coalitions and collaborations wax and wane, driven by both human interests and possibly machine learning. A peer-to-peer policy mechanism of this type invites trepidation amongst those versed in control mechanisms, but it is really no more than a distributed genetic algorithm. With appropriate constraints it could be made to lead to sensible convergent behaviour, or to catastrophically unstable behaviour.

One example of such a collaborative network that has led to positive results is the Open Source Community. The lesson of Open Source Software is that it leads to a rapid evolution. A similar rapid evolution of policy could also be the result from such 


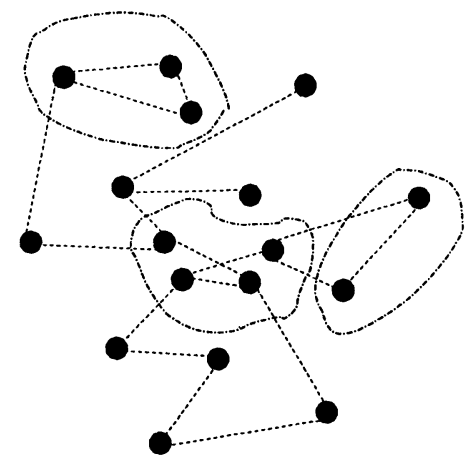

Figure 6 Model 6. Free exchange of policies in a peerto-peer fashion; all nodes have choice (dark). Nodes can form spontaneous, transient coalitions, as indicated by the dashed cells. All nodes can choose; question marks are suppressed.

exchanges. Probably policies would need to be weighted according to an appropriate fitness landscape. They could include things like shared security fixes, best practices, code revisions, new software, and so on. Until this exchange nears a suitable stationary point, policy updates could be much more rapid than for the previous models. This could potentially dominate configuration management behaviour.

This model has no centre. Hence it is, by design, scale-free: all significant interactions are local. Therefore, in principle, if the model can be made to work at small system size, then it will also work at any larger size.

We note however that Model 6, of all the models presented here, has the greatest freedom to explore the space of possible policies. Hence an outstanding, and extremely nontrivial, question for this peer-to-peer model of configuration management is: can such a system find 'better' policies than centralized systems?

\section{Summary and conclusion}

We have presented several models for configuration management on networks. Our Models 3-6 depart from mainstream practice in various ways. The motivation for considering these models is the perception that highly centralized systems are not well adapted to networks that are too large, too heterogeneous, or too dynamic. Since current and future networks are taking on more and more of these three qualities, it is of interest to examine alternative models for configuration management.

We have held ourselves to a limited set of goals. The first of these is the definition of the models themselves. These models offer broad avenues for future research in configuration management; variants of one (or several) of them are likely to be important in future systems.

Our second goal has been to assess the scaling behaviour of these models with respect to two criteria: communication of the current policy to the hosts, and enforcement of the communicated policy. We have considered the various models' ability to meet these criteria, as the number of hosts $N$ in the network grows large. We find, not surprisingly, that the highly centralized systems suffer from a communications bottleneck that limits the size at which they can function effectively. De-centralizing one or both of the two functions gives much better scaling behaviour-to the point that all of the Models 3-6 can, in principle (with some qualifications), implement policy communication and enforcement for very large systems.

Of course, de-centralization brings with it new problems, not addressed by the centralized system: problems of trust, of the quality of chosen policies, and of convergence to a stable regime. These new problems offer attractive issues for further 
research, due both to their intrinsic interest, and to their relevance to the future implementation of de-centralized network systems.

\section{References}

[1] H. Abdu, H. Lutfiya, and M. Bauer. A model for adaptive monitoring configurations. Proceedings of the VI IFIP/IEEE IM conference on network management, page 371, 1999.

[2] M. Burgess. A site configuration engine. Computing systems (MIT Press: Cambridge MA), 8:309, 1995.

[3] M. Burgess. On the theory of system administration. Submitted to J. ACM., 2000.

[4] M. Burgess. Cfengine's immunity model of evolving configuration management. Submitted to IEEE Transactions on Software Engineering, 2002.

[5] M. Burgess. System administration as communication over a noisy channel. Proceedings of the 3nd international system administration and networking conference (SANE2002), 2002.

[6] J. Case, M. Fedor, M. Schoffstall, and J. Davin. The simple network management protocol. RFC1155, STD 16, 1990.

[7] A.L. Couch. Chaos out of order: a simple, scalable file distribution facility for intentionally heterogeneous networks. Proceedings of the Eleventh Systems Administration Conference (LISA XI) (USENIX Association: Berkeley, CA), page 169, 1997.

[8] N. Damianou, N. Dulay, E.C. Lupu, and M. Sloman. Ponder: a language for specifying security and management policies for distributed systems. Imperial College Research Report DoC 2000/1, 2000.

[9] P. Gupta and P.R. Kumar. The capacity of wireless networks. IEEE Trans. Info. Theory, 46(2):388$404,2000$.

[10] J. Li, C. Blake, D.S.J. DeCouto, H.I. Lee, and R. Morris. Capacity of ad hoc wireless networks. Proc. 7th ACM Intl. Conf. on Mobile Computing and Networking, pages 61-69, 2001.

[11] J.P. Martin-Flatin. Push vs. pull in web-based network management. Proceedings of the VI IFIP/IEEE IM conference on network management, page 3, 1999.

[12] M. Matsushita. Telecommunication management network. NTT Review, 3:117-122, 1991.

[13] R. Osterlund. Pikt: Problem informant/killer tool. Proceedings of the Fourteenth Systems Administration Conference (LISA XIV) (USENIX Association: Berkeley, CA), page 147, 2000.

[14] D.B: West. Introduction to Graph Theory (2nd Edition). (Prenctice Hall, Upper Saddle River), 2001.

[15] M. Zapf, K. Herrmann, K. Geihs, and J. Wolfang. Decentralized snmp management with mobile agents. Proceedings of the VI IFIP/IEEE IM conference on network management, page 623, 1999. 